\title{
Participação da sensibilidade atópica em pacientes com ceratoconjuntivite alérgica primaveril
}

\author{
Participation of atopic sensitivity in patients with vernalallergickeratoconjunctivitis
}

\author{
Denise Atique Goulart ${ }^{1}$ \\ Débora Marcolini Schneider ${ }^{2}$ \\ Evely Tanaka ${ }^{3}$ \\ Maria da Conceição Menezes Santos ${ }^{3}$ \\ Maria Cristina Nishiwaki-Dantas ${ }^{4}$ \\ Wilma Carvalho Neves Forte ${ }^{5}$
}

\begin{tabular}{l} 
RESUMO \\
\hline Objetivos: Estudar as respostas dos testes de punctura cutâneos nos \\
pacientes portadores de ceratoconjuntivite vernal e comparar as respostas \\
com as da população alérgica sem acometimento ocular. Métodos: Foram \\
realizados testes alérgicos de punctura em 48 pacientes portadores de \\
ceratoconjuntivite alérgica primaveril, sendo 10 da forma limbar, 19 da \\
forma palpebral e 19 da forma mista. O grupo controle foi formado por 52 \\
pacientes do ambulatório de alergia da Santa Casa de São Paulo, portado- \\
res de alergia sistêmica, porém sem acometimento ocular. Testes cutâneos \\
de punctura foram realizados em 48 pacientes do Setor de Córnea e \\
Doenças Externas Oculares, do Ambulatório de Alergia Ocular do Depar- \\
tamento de Oftalmologia da Santa Casa de São Paulo, no período de julho \\
de 2001 a setembro de 2002. O indicativo de resposta alérgica positiva era \\
reação cutânea maior que 2 mm ocorrendo até 15 minutos da aplicação do \\
alérgeno. Foram avaliadas as respostas aos seguintes alérgenos: pó \\
doméstico, Dermatophagoides pteronissynus, Dermatophagoides fari- \\
nae, Tyrophagus putrescentiae, Blomia tropicalis, Rhizopus spp, Peni- \\
cillium, Alternaria alternata e pólens de gramíneas, disponíveis naquele \\
serviço no dado período. Resultados: Na população estudada, o sexo \\
masculino foi mais freqüente, com 32 pacientes (66,6\%) no grupo de estudo \\
e 27 no controle (51,9\%). A idade média foi $10,0 \pm 4$ anos no grupo de \\
estudo e 6,83 $\pm 3,6$ anos no grupo controle. O alérgeno mais comumente \\
causador de crises nos pacientes foi Dermatophagoides pteronissynus, \\
compositividade em 26 pacientes, seguidopor Dermatophagoidesfarinae, \\
com 24 pacientes, Blomia tropicalis com 22 , pó doméstico com $17, T$. \\
putrescentiae com 15, e Rhizopus com 3 . Os alérgenos Penicillium, \\
Alternaria alternata, e pólen de gramíneas tiveram cada um apenas 1 \\
paciente com positividade no grupo com CCP. Conclusões: A semelhança \\
do resultado do teste nos pacientes sem acometimento ocular nos indica \\
que a conjuntivite alérgica nada mais é que uma das formas de expressão \\
da atopia, assim como são a rinite, a bronquite e a dermatite. \\
\hline
\end{tabular}

Descritores: Conjuntivite alérgica; Alérgenos/uso diagnóstico; Córnea/patologia; Córnea/ imunologia; Diagnóstico diferencial; Testes cutâneos

\section{INTRODUÇÃO}

As alergias oculares são doenças freqüentes e representam uma importante porcentagem das consultas em clínicas oftalmológicas ${ }^{(1)}$. A ceratoconjuntivite primaveril (CCP) é uma afecção alérgica recorrente e bilateral, geralmente grave, que acomete, principalmente, meninos em idade pré- 
escolar e escolar que se queixam de prurido intenso, lacrimejamento, sensação de corpo estranho, fotofobia e embaçamento visual $^{(2)}$. Ao exame, apresentam edema palpebral, injeção conjuntival, secreção mucosa esbranquiçada entre as papilas hipertróficas, que podem fundir-se formando as papilas gigantes na conjuntiva palpebral superior (forma palpebral). Hipertrofia papilar também pode ser encontrada no limbo córneoescleral (forma límbica), conferindo-lhe aspecto "gelatinoso" e, geralmente, ocorre em pacientes de origem não caucasiana, principalmente negros. O acometimento corneal caracteriza-se por ceratite punctata superficial que pode evoluir para úlcera de córnea chamada também de "úlcera em escudo" pelo aspecto clínico típico de pacientes com conjuntivite primaveril. Todas as formas de conjuntivite alérgica primaveril se resolvem espontaneamente quando os pacientes atingem a puberdade ou idade adulta ${ }^{(3-4)}$.

Doença comum em países tropicais como o Brasil, a conjuntivite primaveril muitas vezes evolui com complicações graves e de difícil controle, que levam à deficiência visual permanente. Tais complicações são responsáveis pela má qualidade de vida de seus portadores e podem ser minimizadas com diagnóstico e tratamento adequados ${ }^{(5-6)}$.

A fisiopatologia desta doença envolve atividade eosinofílica e produção de citocinas. A primeira etapa do mecanismo imunológico envolve atividade das células de Langerhans, que apresentam o antígeno que interage com o linfócito $\mathrm{T}$ helper. As interleucinas liberadas por ele estimulam o linfócito B que sofrerá desvio na produção de IgG para produção de IgE. Numa segunda etapa, a IgE entra em contato com os anticorpos das membranas dos mastócitos e há degranulação, com liberação de mediadores vasoativos, enzimas e mediadores químicos, como o fator quimiotático eosinofílico ${ }^{(7)}$.

O diagnóstico da CCP é basicamente clínico, no entanto, nas fases iniciais, seus sintomas são frustros podendo ser confundida com outras doenças oculares, como a blefarite anterior e posterior, o que retarda o tratamento ${ }^{(8)}$. A confirmação diagnóstica pode ser feita por meio da citologia esfoliativa para a conjuntiva, e o corante mais utilizado é o Giemsa, que evidencia tipos celulares característicos desta doença, como a presença de eosinófilos e grânulos eosinofílicos, neutrófilos etc ${ }^{(9)}$.

O termo atopia é usado para descrever a reação alérgica em indivíduos com predisposição genética a doenças que demonstrem positividade na reação de Prausnitz- Kustner. A existência de atopia, encontrada em $15 \%$ da população geral, indica doença alérgica e predisposição a doenças como as conjuntivites alérgicas ${ }^{(10)}$.

O teste alérgico de punctura (Prick Test) é um método seguro, de fácil realização e de baixo custo utilizado para detecção dos alérgenos envolvidos na sensibilização de pacientes atópicos. Este teste é amplamente utilizado pelos clínicos alergistas e em pacientes com alergia ocular pode ajudar a evitar crises, já que permite que o paciente evite o contato com os alérgenos aos quais é sensível ${ }^{(8)}$.

\section{OBJETIVO}

O objetivo do presente estudo foi observar o resultado do teste alérgico de punctura (Prick Test) em pacientes portadores de diferentes diagnósticos clínicos de ceratoconjuntivite alérgica primaveril e comparar os resultados com uma população alérgica livre de acometimento ocular.

\section{MÉTODOS}

Foram realizados testes alérgicos de punctura (Prick Test) em 48 pacientes portadores de ceratoconjuntivite primaveril (CCP) do Setor de Córnea e Doenças Externas - Ambulatório de Alergia Ocular da Santa Casa de São Paulo, no período de julho de 2001 a setembro de 2002. Dez pacientes apresentavam a forma límbica, 19 a forma palpebral e 19 a forma mista da CCP.

No grupo controle, 52 pacientes portadores de alergia sistêmica com diagnósticos diversos (rinite, asma brônquica e dermatite), porém sem acometimento ocular, foram submetidos ao teste alérgico de punctura (Prick Test).

Os testes foram feitos com aplicação de uma gota do alérgeno no antebraço das crianças seguida de laceração superficial da pele com agulha fina. Os extratos usados foram: pó doméstico, Dermatophagoides pteronissynus, Dermatophagoides farinae, Tyrophagus putrescentiae, Blomia tropicalis, Rhizopus spp, penicilium, Alternaria alternata, Periplaneta americana, Blatella germanica, pólens de gramíneas, disponíveis no Ambulatório de Imunologia Pediátrica da Santa Casa de Misericórdia de São Paulo naquele momento. Outros extratos, como cão e gato, não estavam disponíveis e por esta razão não foram usados. A histamina foi usada como controle positivo $(1 \mathrm{mg} / \mathrm{ml})$ e o diluente (solução salina tamponada com albumina humana $0,03 \%$ ) como controle negativo. $O$ indicativo de resposta alérgica positiva foi reação cutânea elevada e hiperemiada, maior que $2 \mathrm{~mm}$, ocorrida até $15 \mathrm{minu}$ tos após a aplicação do alérgeno ${ }^{(11)}$. O grau de positividade era dado pela comparação do tamanho, elevação e hiperemia com o controle positivo e graduado em cruzes de + a $5+$. Foi considerada resposta positiva a resposta a partir de $1+$. O teste foi realizado por pediatras experientes do Ambulatório de Alergia e Imunologia Pediátrica da Santa Casa de São Paulo.

A análise estatística foi feita por meio do teste do quiquadrado, teste exato de Fisher e t de Student para amostras independentes. O nível de significância utilizado foi de 5\%.

\section{RESULTADOS}

Trinta e dois $(66,6 \%)$ pacientes do grupo de estudo eram do sexo masculino e $16(33,4 \%)$ eram do sexo feminino. Quanto ao grupo controle, 27 pacientes $(51,9 \%)$ eram do sexo masculino e $25(48,1 \%)$, do feminino.

A idade média do grupo de estudo foi de 10 anos $( \pm 4,7)$ e do grupo controle foi de 6,8 anos $( \pm 3,6)$. 
De todos os pacientes com CCP testados, 38 (76\%) obtiveram reação positiva a algum tipo de alérgeno no teste alérgico de punctura (Prick Test) e 12 (24\%) obtiveram resultado negativo no teste.

Todos os pacientes do grupo controle apresentaram algum tipo de positividade ao teste alérgico de punctura (Prick Test).

Os resultados do teste alérgico de punctura (Prick Test) estão dispostos na tabela 1 .

\section{DISCUSSÃO}

A CCP é uma das variantes mais graves das conjuntivites alérgicas e, portanto deve ser diagnosticada e tratada apropriadamente. No Ambulatório de Alergia Ocular da Santa Casa de São Paulo esta forma de alergia ocular corresponde a mais da metade de todos os $\operatorname{casos}^{(10)}$ e observamos, na prática diária, que muitos dos pacientes estudados apresentam não só o acometimento oftalmológico, mas também, sinais e sintomas de alergia sistêmica.

Os resultados dos testes alérgicos de punctura (Prick Tests) revelaram que a maior parte dos nossos pacientes do grupo de estudo (76\%) apresentaram reação positiva a algum tipo de alérgeno testado, número um pouco maior do que aqueles já relatados na literatura ${ }^{(8,11)}$. Este alto índice de positividade nos nossos pacientes demonstra que em sua maioria o tipo de hipersensibilidade envolvida na gênese de suas alergias é a reação mediada por Ig E (tipo I). Por outro lado, a existência de pacientes com o resultado negativo do teste fortalece a hipótese de que as alterações imunológicas envolvidas na fisiopatologia da CCP são mais complexas e, prova-

\begin{tabular}{|c|c|c|c|c|}
\hline Alérgeno & Categoria & $\begin{array}{l}\text { Grupo } \\
\text { Estudo }\end{array}$ & $\begin{array}{l}\text { Grupo } \\
\text { Controle }\end{array}$ & p \\
\hline Pó doméstico & $\begin{array}{l}- \\
+\end{array}$ & $\begin{array}{ll}31 & (64,6 \%) \\
17 & (35,4 \%)\end{array}$ & $\begin{array}{l}35(67,3 \%) \\
17(32,7 \%)\end{array}$ & 0,774 \\
\hline D. pteronissynus & + & $\begin{array}{l}22(45,8 \%) \\
26(54,2 \%)\end{array}$ & $\begin{array}{ll}20 & (38,5 \%) \\
32 & (61,5 \%)\end{array}$ & 0,456 \\
\hline D. farinae & + & $\begin{array}{l}24(50,0 \%) \\
24(50,0 \%)\end{array}$ & $\begin{array}{ll}24 & (46,2 \%) \\
28 & (53,8 \%)\end{array}$ & 0,701 \\
\hline T. putrescentiae & + & $\begin{array}{l}33(68,8 \%) \\
15(31,3 \%)\end{array}$ & $\begin{array}{l}39(75,0 \%) \\
13(25,0 \%)\end{array}$ & 0,487 \\
\hline Blomia tropicalis & + & $\begin{array}{l}26(54,2 \%) \\
22(45,8 \%)\end{array}$ & $\begin{array}{ll}25 & (48,1 \%) \\
27 & (51,9 \%)\end{array}$ & 0,543 \\
\hline Rhizopus & + & $\begin{array}{r}45(98,3 \%) \\
3(6,3 \%)\end{array}$ & $\begin{array}{r}50(96,2 \%) \\
2(3,8 \%)\end{array}$ & $0,669^{\#}$ \\
\hline Penicillium & + & $\begin{array}{r}46(95,8 \%) \\
2(\mathbf{4 , 2 \% )}\end{array}$ & $\begin{array}{c}52(100,0 \%) \\
0\end{array}$ & $0,228^{\#}$ \\
\hline Alternaria & $\begin{array}{l}- \\
+\end{array}$ & $\begin{array}{r}44(91,7 \%) \\
4(\mathbf{8 , 3 \% )}\end{array}$ & $\begin{array}{c}52(100,0 \%) \\
0\end{array}$ & $0,05^{\#}$ \\
\hline Pólens de gramíneas & as - & $\begin{array}{r}47(97,9 \%) \\
1(2,1 \%)\end{array}$ & $\begin{array}{r}49(94,2 \%) \\
3(5,8 \%)\end{array}$ & $0,619^{\#}$ \\
\hline
\end{tabular}

velmente, envolvem tanto a hipersensibilidade do tipo I quanto à mediada por células (tipo IV) ${ }^{(12)}$.

Os extratos relacionados ao pó e ácaro domésticos $(D$. pteronissynus, $D$. farinae) foram os mais frequientemente positivos, fato que enfatiza a importância da higiene ambiental como parte do tratamento destes pacientes. Durante a consulta, o médico oftalmologista deve orientar mudanças de hábitos, tais como: retirar carpetes do quarto, lavar as roupas de cama com água quente várias vezes por mês, usar travesseiro de látex, manter a casa ventilada, não criar animais e não fumar, além do tratamento médico específico.

\section{CONCLUS ÃO}

A semelhança do resultado do teste nos pacientes estudados com ou sem acometimento ocular evidencia a possibilidade de que a conjuntivite alérgica pode ser uma das formas de expressão da atopia, assim como são a rinite, a bronquite e a dermatite. No entanto, sugerimos continuação deste estudo para resultados mais precisos.

\section{AGR ADECIMENTOS}

Ao Laboratório de Imunologia da Santa Casa de São Paulo.

\section{ABSTRACT}

Purpose: To study the response to skin prick tests in patients with vernal keratoconjunctivitis and compare with the allergic population without ocular disease. Methods: We performed skin prick tests in 48 patients with VKC (10 with limbal form, 19 with palpebral and 19 with both). Controls were 52 patients from Allergy Clinic of the Santa Casa de São Paulo, with systemic allergy but without ocular disease. Skin prick tests were performed in 48 patients from the Ocular Allergy Sector at the Ophthalmology Department of the Santa Casa de São Paulo's Hospital, from July 2001 to September 2002. We studied responses to the following allergens: house dust mite, Dermatophagoides pteronissynus, Dermatophagoides farinae, Tyrophagus putrescentiae, Blomia tropicalis, Rhizopus spp, Penicilium, Alternaria alternata and rye grass. Results: Male sex was the most frequent, with 32 patients $(66.6 \%)$ in the study group, and 27 (51.9\%) in controls. Mean age was $10.0 \pm$ 4.7 years in the study group and $6.83 \pm 3.6$ in controls. The most frequent allergen was Dermatophagoides pteronissynus, followed by Dermatophagoides farinae, Blomia tropicalis, house dust mite, Tyrophagus putrescentiae and Rhizopus spp. Conclusions: The resemblance of the results in the control group indicates that allergic conjunctivitis is nothing else but another form of atopy, just as rhinitis, bronchitis and dermatitis.

Keywords: Conjunctivitis, allergic; Allergens/diagnostic use; Cornea/pathology; Cornea/immunology; Differential diagnosis; Skin tests 


\section{REFERÊNCIAS}

1. Höfling-Lima AL, Andrade AJM, Marback PMF, Farah ME, Mascaro V. Comparação do uso tópico do cetotifeno com a olopatadina no tratamento das conjuntivites alérgicas. Arq Bras Oftalmol 2001;64:415-22.

2. Sena CM, Tanure MA, Cruz ACG, Trindade F, Pereira FAS. Uso da medicação homeopática no tratamento da ceratoconjuntivite primaveril - resultados iniciais. Arq Bras Oftalmol 2003;66:45-50.

3. Abelson MB, George MA, Garofalo C. Differential diagnosis of ocular allergic disorders [published erratum in Ann Allergy 1993;70:192]. Ann Allergy 1993;70:95-109.

4. Bonini S, Lambiase A, Bonini S. Genetic and environmental factors in ocular allergy. In: Abelson MB, Allergic diseases of the eyes. Philadelphia: WB Sanders; 2000. p.1-10.

5. Shuler JD, Levenson J, Mondino BJ. Inferior corneal ulcers associated with palpebral vernal conjunctivitis. Am J Ophthalmol 1988;106:106-7.

6. Trocmé SD, Kephart BS, Bourne WM, Buckley RJ, Gleich GJ. Eosinophil granule major basic protein deposition in corneal ulcers associated with vernal keratoconjunctivitis. Am J Ophthalmol 1993;115:640-3.
7. Botelho PBM, Marback PMF, Sousa LB, Campos M, Vieira LA. Ceratoconjuntivite alérgica e complicações no segmento anterior de pacientes. Arq Bras Oftalmol 2003;66:25-8.

8. Bousquet J. In view methods for study allergy. In: Adkinson NF, Busse W, Bochner B, Stephen H, Elliot M, Yunginger JW, editors. Middleton's allergy: principles and practice. $6^{\text {th }}$ ed. St. Louis. Mosby; 2003. p.427-34.

9. Bezerra HL, Rizzo LV, Yu MCZ, Freitas D. Avaliação da resposta terapêutica nas alergias oculares por meio da citologia esfoliativa. Arq Bras Oftalmol 2003;66:595-8.

10. Goulart DA, Goulart DG, Cypel MC, Dantas PEC, Nishiwaki-Dantas MC Perfil clínico-epidemiológico de pacientes do Ambulatório de Alergia Ocular da Santa Casa de São Paulo. Arq Bras Oftalmol 2003;66:609-18.

11. Bonini S, Bonini S, Lambiase A, Marchi S, Pasqualetti P, Zuccaro O, et al. Vernal keratoconjunctivitis: revisited a case series of 195 patients with longterm follow up. Ophthalmology 2000;107:1157-63.

12. Dalens H, Fauquert JL, Chatron P, Beaujon G. Vernal keratoconjuntivitis in the child. Clinical and complementary study a propos of 22 cases. J Fr Ophthalmol 1998;21:471-8.

\title{
XIV Congresso \\ da Sociedade Norte-Nordeste \\ de Oftalmologia
}

\section{0 de Junho a 3 de Julho de 2004 \\ Rio Poti Hotel - Teresina - PI}

\author{
Informações: Agenda Promoções Ltda. \\ Fone/ Fax: (86) 223-9444 \\ E-mail: oftalmologia.nortenordeste.2004@bol.com.br \\ Home-page: www.agendapromocoes.com.br
}

